\title{
HALLAZGO DE Lophomonas sp. EN SECRECIONES DEL TRACTO RESPIRATORIO DE NIÑOS HOSPITALIZADOS CON ENFERMEDAD PULMONAR GRAVE
}

\author{
Rito Zerpa $a^{1,2, a}$, Elsa Ore ${ }^{1, b}$, Lilian Patiño $0^{1, a}$, Yrma A. Espinoza ${ }^{2, c}$
}

\begin{abstract}
RESUMEN
Lophomonas $s p$. es un parásito habitual del tracto intestinal de las cucarachas y que no es reconocido como patógeno humano. Sin embargo, en la literatura mundial existen escasos reportes de Lophomonas sp. en secreciones del tracto respiratorio en pacientes con enfermedad pulmonar grave, principalmente en adultos. Presentamos evidencias de Lophomonas sp. en el tracto respiratorio inferior de niños atendidos en el centro de referencia nacional de enfermedades pediátricas de Lima, Perú, en el periodo 2009-2010. Se encontró seis casos, 4/23 provenientes de muestras de lavado broncoalveolar y 2/794 muestras de aspirado traqueal de niños. Cinco de ellos tuvieron neumonía y uno atelectasia, cuatro estuvieron en la unidad de cuidados intensivos. Es necesario conocer más sobre la presencia de este organismo en infecciones respiratorias así como su rol patogénico real.
\end{abstract}

Palabras clave: Infecciones por protozoarios; Enfermedades pulmonares parasitarias; Lavado broncoalveolar (fuente: DeCS BIREME).

\section{Lophomonas sp. IN RESPIRATORY TRACT SECRETIONS IN HOSPITALIZED CHILDREN WITH SEVERE LUNG DISEASE}

\begin{abstract}
Lophomonas $s p$. is a habitual parasite of the intestinal tract of the cockroaches and that is not recognized as pathogenic human being. Nevertheless, in the world literature are few reports of Lophomonas sp. in respiratory tract secretions in patients with severe pulmonary disease, mostly in adults. We present evidences of Lophomonas sp. in the respiratory low tract of children attended in the national reference center of paediatric diseases of Lima, Peru, in the period 20092010. We found six cases, $4 / 23$ from broncoalveolar lavage and 2/794 from tracheal aspirate samples of children. Five of them had pneumonia and one atelectasis, four were hospitalized in the intensive care unit. Increase knowledge about the presence of this organism in respiratory infections is needed, as its real pathogenic role.
\end{abstract}

Key words: Protozoan infections; Lung diseases, parasitic; Bronchoalveolar lavage (source: MeSH NLM).

\section{INTRODUCCIÓN}

Las infecciones pulmonares causadas por protozoarios adquieren cada vez mayor interés debido a que se presentan en pacientes inmunocomprometidos entre ellos los transplantados, entre quienes usan corticoides, con SIDA, tener neoplasias, entre otros ${ }^{(1,2)}$. Aunque se describen generalmente como reportes de caso, su presentación es cada vez más frecuente; entre los protozoarios implicados se encuentran patógenos que suelen atacar otros órganos y que esporádicamente afectan las vías respiratorias como: Entamoeba histolytica, Acanthamoeba castellani, Naegleria fowleri, Balamuthia mandrillaris, Trichomonas vaginalis, Leishmania sp, Trypanosoma cruzi, Cyclospora cayetanensis, Cryptosporidium parvum, Toxoplasma gondii, Plasmodium falciparum, Babesia microti y Balantidium coli (2).

El Lophomonas blattarum es un parásito habitual de la flora intestinal de las cucarachas (Periplaneta ameri- cana); en China se halló en muestras de secreciones respiratorias de pacientes adultos con neumonías bacterianas que no responden a tratamiento ${ }^{(3-6)}$. También ha sido encontrado en muestras de esputo de pacientes asmáticos y algunos consideran que puede tener alguna implicancia en la recurrencia de crisis en pacientes asmáticos ${ }^{(7,8)}$.

En año 2002, encontramos cuatro casos de Lophomonas $\mathrm{sp}$. al evaluar técnicas para mejorar la visualización de protozoos en muestras de secreciones respiratorias, en niños hospitalizados en el Instituto Nacional de Salud del Niño en Lima, Perú ${ }^{(9)}$.

Por ello, el propósito de este estudio es evaluar la presencia de Lophomonas en muestras de secreciones respiratorias en niños hospitalizados en el Instituto Nacional de Salud del Niño, durante el 2009 y 2010.

Servicio de Microbiología, Instituto Nacional de Salud del Niño, Lima, Perú

Instituto de Medicina Tropical "Daniel A. Carrión”, Universidad Nacional Mayor de San Marcos, Lima, Perú.

Médico Patólogo Clínico; ${ }^{\mathrm{b}}$ Tecnólogo Médico; ' Bióloga.

Recibido: 03-11-10 Aprobado: 01-12-10 
Tabla 1. Pacientes con hallazgo de Lophomonas sp. en secreciones del tracto respiratorio inferior.

\begin{tabular}{|c|c|c|c|c|c|}
\hline Caso & Edad & Procedencia & Hospitalización & Diagnóstico & Muestra \\
\hline 1 & 8 años & Lima & $\mathrm{UCl}$ & Sepsis, neumonía & Aspirado traqueal \\
\hline 2 & 15 años & Lima & Medicina & Neumonía necrotizante cavitada. & Lavado broncoalveolar \\
\hline 3 & 18 meses & Ayacucho & Cardiología & $\begin{array}{l}\text { Sepsis, cardiopatía congénita, } \\
\text { bronconeumonía, neumonía } \\
\text { asociado a ventilador, desnutrición } \\
\text { crónica. }\end{array}$ & Aspirado traqueal \\
\hline 4 & 4 meses & Lima & $\mathrm{UCl}$ & Neumonía & Lavado broncoalveolar \\
\hline 5 & 4 meses & Piura & $\mathrm{UCl}$ & $\begin{array}{l}\text { Cardiopatía congénita, atelectasia } \\
\text { basal, síndrome de Down. }\end{array}$ & Aspirado traqueal \\
\hline 6 & 5 años & Lima & $\mathrm{UCl}$ & Neumonía & Aspirado traqueal \\
\hline
\end{tabular}

UCl: Unidad de cuidados intensivos.

\section{EL ESTUDIO}

Se realizó un estudio descriptivo, transversal durante enero de 2009 y julio de 2010 en el Servicio de Microbiología del Instituto Nacional de Salud del Niño, que es el centro de referencia nacional del Perú para enfermedades pediátricas.

Se incluyó todas las muestras del tracto respiratorio inferior (aspirado traqueal y lavado broncoalveolar) que llegaron para diagnóstico microbiológico. Todas las muestras fueron procesadas en montaje húmedo con solución salina para la detección de Lophomonas $s p$, observadas con objetivos de 10X y 40X, se registró las imágenes en fotomicrografías ${ }^{(9,10)}$. No se pudo tener acceso a las historias clínicas de los casos positivos.

\section{HALLAZGOS}

Se encontró Lophomonas sp. en 4/23 muestras de lavado broncoalveolar y en 2/794 de aspirado traqueal, cuatro niños provenían de la Unidad de Cuidados Intensivos, uno del servicio de cardiología y uno del servicio de medicina. Todos presentaron enfermedad pulmonar grave, cinco presentaron neumonía y uno atelectasia
(Tabla 1). Las características de Lophomonas sp. se presentan en fotomicrografías (Figuras 1 y 2).

\section{DISCUSIÓN}

Los resultados de este estudio, aunque preliminares, demuestran la presencia de Lophomonas $s p$. en niños hospitalizados con enfermedad respiratoria grave en el principal hospital pediátrico del Perú. No se encontró reportes previos de Lophomonas en Latinoamérica publicados en revistas científicas (búsquedas realizadas en LILACS, SciELO, MEDLINE y Scholar Google), el único antecedente es la comunicación realizada en una jornada científica el año $2002^{(9,10)}$.

Los escasos reportes de Lophomonas infectando humanos, han sido en adultos con inmunosupresión o enfermedad pulmonar grave ${ }^{(2-6)}$, nuestros hallazgos en niños indican la presencia del parásito y su importancia en infecciones del tracto respiratorio inferior en forma similar a lo observado en adultos.

Las Lophomonas, que son parásitos habituales de la flora intestinal de las cucarachas, y se han encontrado an-

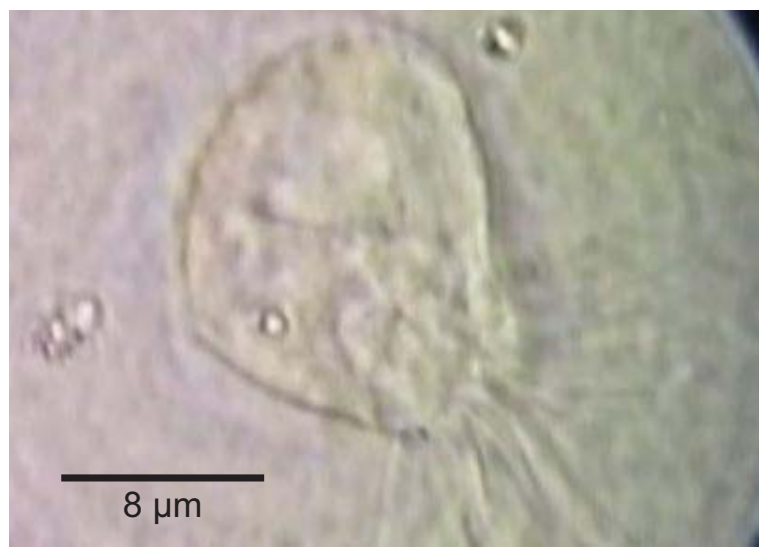

Figura 1. Lophomonas sp. en muestra de aspirado traqueal, preparación en montaje húmedo. 


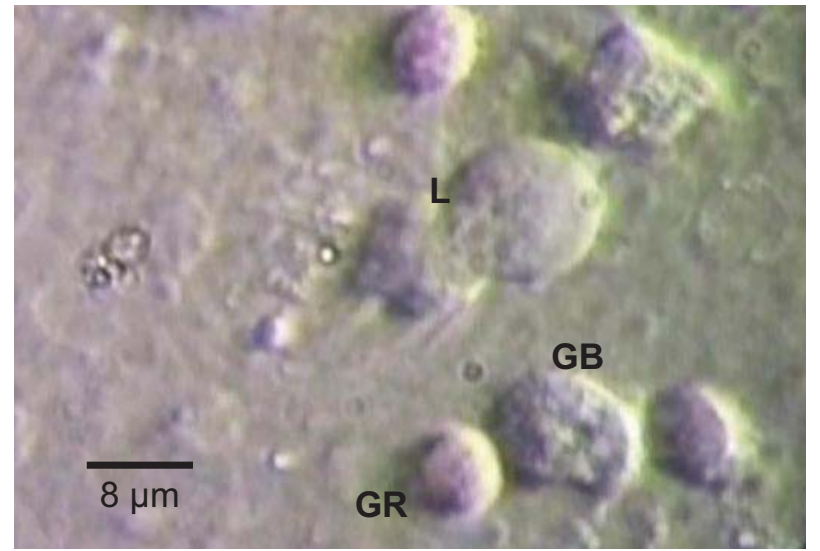

Figura 2. Lophomonas sp. (L) entre leucocitos (GB) y hematíes (GR), en muestra de aspirado traqueal; preparación en montaje húmedo.

tes en el país ${ }^{(11)}$, no estaban asociadas con enfermedad humana, sin embargo, debido a los últimos hallazgos, algunos autores la sindican como patógeno pulmonar ${ }^{(12)}$, ya que también ha sido encontrada en muestras de esputo en pacientes asmáticos ${ }^{(7)}$. El manejo de estos casos es con metronidazol según han demostrado los reportes previos ${ }^{(2,13)}$.

Todavía falta mucho por estudiar sobre el potencial patógeno de este protozoario, como su frecuencia en pacientes con enfermedad respiratoria grave en la que no se encuentran patógenos conocidos, así como en pacientes asmáticos.

Al no ser considerado previamente como patógeno, cuando se encuentran protozoos flagelados en secreciones respiratorias no pensamos en Lophomonas y menos que esta sea la causa de la enfermedad, por lo que no suele reportarse en los informes, además las técnicas convencionalmente usadas como el Gram no permiten su adecuada visualización ${ }^{(9)}$. Por ello, en los laboratorios de los hospitales de mayor nivel, debería realizarse el montaje húmedo con solución salina para buscar este protozoario.

Entre las limitaciones de este trabajo, se encuentra la falta de acceso a las historias clínicas lo que no nos permitió una mejor descripción de cada uno de los casos. Las muestras de secreciones respiratorias que llegaron al Servicio de Microbiología fueron a demanda y a criterio de cada clínico, por lo que no podemos establecer una frecuencia de la enfermedad en nuestro hospital. No se pudo identificar la especie de Lophomonas.

En conclusión, demostramos la presencia de Lophomonas $s p$. en secreciones respiratorias de niños hospitalizados en el hospital pediátrico de referencia nacional en Lima, Perú; recomendamos tener en cuenta a este pro- tozoario en el diagnóstico diferencial de pacientes con infección pulmonar grave sin etiología conocida.

\section{Financiamiento}

Autofinanciado

\section{Conflictos de Interés}

Los autores declaran no tener conflictos de interés en la publicación de este artículo.

\section{REFERENCIAS BIBLIOGRÁFICAS}

1. Vijayan VK. Parasitic lung infection. Curr Opin Pulm Med. 2009;15(3):274-82.

2. Martinez-Girón R, Esteban JG, Ribas A, Doganci L. Protozoa in respiratory pathology: a review. Eur Respir J. 2008;32(5):1354-70.

3. Wang Y, Tang Z, Ji S, Zhang Z, Chen J, Cheng Z, et al. Pulmonary Lophomonas blattarum infection in patients with kidney allograft transplantation. Transpl Int. 2006;19(12):1006-13.

4. Yao GZ, Zeng LQ, Zhang B, Chang ZS. [Bronchopulmonary Lophomonas blattarum infection: two cases report and literature review]. Zhonghua Nei Ke Za Zhi. 2008;47(8):63437. [artículo en chino]

5. Shi YL, Li LH, Liao Y, Li XN, Huang XY, Liu J, et al. [Diagnosis and treatment of Lophomonas blattarum infection in 26 patients with bacterial pneumonia]. Zhongguo Ji Sheng Chong Xue Yu Ji Sheng Chong Bing Za Zhi. 2007;25(5):43031. [artículo en chino]

6. Yao G, Zhou B, Zeng L. Imaging characteristics of bronchopulmonary Lophomonas blattarum infection: case report and literature review. J Thorac Imaging. 2009;24(1):49-51.

7. Martínez-Girón R, Ribas A, Astudillo-González A. Flagellated protozoa in cockroaches and sputum: the unhygienic connection? Allergy Asthma Proc. 2007;28(5):608-9.

8. Martínez-Girón R, Ribas A. Asthma, cockroaches, and protozoal forms: chance or not chance? Ann Allergy Asthma Immunol. 2006;97(6):818-19.

9. Zerpa R, Huiza A, Espinoza Y, Ore E. Utilidad del montaje húmedo para la detección de Lophomonas $\mathrm{sp}$. en secreciones respiratorias. An Fac Med (Lima). 2004;65(Supl):24.

10. Zerpa R, Huiza A, Espinoza Y, Ore E. Lophomonas sp.: una nueva visión. An Fac Med (Lima). 2004;65(Supl):22.

11. Fernández M, Martínez DM, Tantaleán M, Martínez R. Parásitos presentes en Periplaneta americana Linnaeus "cucaracha doméstica" de la ciudad de Ica. Rev Peru Biol. 2001;8(2):105-13.

12. Martínez-Girón R, Doganci L. Lophomonas blattarum: a brochopulmonary pathogen. Acta Cytol. 2010;54(Suppl):1050-51.

13. Ribas A, Martínez-Girón R, Ponte-Mittelbrum C, AlonsoCuervo R, Iglesias-Llaca F. Immunosupression, flagellated protozoa in the human airways and metronidazole: observations on the state of the art. Transpl Int. 2007;20(9):811-12.

Correspondencia: Rito Zerpa Larrauri

Correo electrónico: rzerpa43@yahoo.com 